\title{
Bronchiolitis obliterans organizing pneumonia after radiation therapy for lung cancer: a case report
}

\author{
Lorenzo Falcinelli ${ }^{1}$, Rita Bellavita ${ }^{1}$, Alberto Rebonato ${ }^{2}$, Rita Chiari ${ }^{3}$, Jacopo Vannucci ${ }^{4}$, Francesco Puma ${ }^{4}$, Cynthia Aristei ${ }^{5}$ \\ ${ }^{1}$ Radiation Oncology Division, Perugia General Hospital, Perugia - Italy \\ ${ }^{2}$ Radiology Section, Department of Surgical and Biomedical Sciences, University of Perugia and Perugia General Hospital, Perugia - Italy \\ ${ }^{3}$ Medical Oncology Section, Perugia General Hospital, Perugia - Italy \\ ${ }^{4}$ Thoracic Surgery Unit, Department of Surgical and Biomedical Sciences, University of Perugia and Perugia General Hospital, Perugia - Italy \\ ${ }^{5}$ Radiation Oncology Section, Department of Surgical and Biomedical Sciences, University of Perugia and Perugia General Hospital, Perugia - \\ Italy
}

\begin{abstract}
Bronchiolitis obliterans organizing pneumonia (BOOP), also known as cryptogenic organizing pneumonia, has mainly been described in patients with breast cancer who received radiotherapy after breast-conserving surgery. In this rare case, a 70-year-old man with left apical squamous lung cancer developed BOOP after radiotherapy and only one cycle of concomitant chemotherapy.

This case report draws attention to the development of this syndrome in the unusual setting of lung cancer, advising prompt steroid treatment when diagnostic images reveal the characteristic signs of the disease.

Keywords: Bronchiolitis obliterans organizing pneumonia, Cryptogenic organizing pneumonia, Interstitial lung disease, Lung cancer, Radiation therapy, Side effects
\end{abstract}

\section{Introduction}

Bronchiolitis obliterans organizing pneumonia (BOOP), or cryptogenic organizing pneumonia, has been almost exclusively reported after radiotherapy (RT) in a very small minority of women who received breast-conserving surgery $(1,2)$. It is characterized by respiratory symptoms such as flu-like illness with or without cough and bronchial crackles $(3,4)$ and peculiar radiographic lesions such as patchy and groundglass opacity extending to nonirradiated parenchyma $(1,5$, 6). Polypoid fibrino-inflammatory granulation tissue is also observed and eosinophil, neutrophil, and lymphocyte counts are increased in bronchoalveolar lavage fluid.

Although BOOP is unusual in patients with lung cancer who receive RT (7), it has been observed after chemotherapy or treatment with targeted therapies (8). Here we describe a rare case of BOOP in a patient with lung cancer with onset after RT and only one cycle of concurrent chemotherapy.

Accepted: September 9, 2014

Published online: April 3, 2015

Corresponding author:

Lorenzo Falcinelli

Radiation Oncology Division

Perugia General Hospital

Perugia, Italy

falcinellil@libero.it

\section{Case report}

A 70-year-old man with a long history of smoking (15-20 cigarettes daily for 50 years) developed left arm pain that did not respond to analgesics. His performance status was good ( $90 \%$ according to Karnofsky) and he had no relevant comorbidities. After 4 months, a chest X-ray showed an opaque area in the upper left field and a computed tomography (CT) scan detected a 4-cm lesion at the left lung apex, infiltrating the clavicle. Disease extent was confirmed by positron emission tomography/CT (PET-CT). Since there was no radiographic or metabolic evidence of pathologic lymphadenopathy, the disease stage was cT4NOMO.

Spirometry showed that $\mathrm{FEV}_{1}$ was $78 \%$ and hemogas analysis indicated a high $\mathrm{pH}$, normal $\mathrm{pCO}_{2}$, and high bicarbonates $\left(\mathrm{pH}=7.45, \mathrm{pCO} 2=37.5 \mathrm{~mm} \mathrm{Hg}, \mathrm{HCO}_{3}^{-}=26.4 \mathrm{mmol} / \mathrm{L}\right)$, suggesting metabolic alkalosis due to respiratory distress.

Biopsy findings in a sample obtained during bronchoscopy indicated squamous carcinoma (cytokeratine 7+, TTF1-, napsine-, $\mathrm{P} 63+$ ).

As the disease was nonoperable, neoadjuvant treatment was started with RT and concurrent chemotherapy (cisplatin $80 \mathrm{mg} / \mathrm{mq}$ and etoposide $80 \mathrm{mg} / \mathrm{mq}$ every 28 days). Only 1 cycle of chemotherapy was administered because of acute grade 3 fatigue and grade 2 thrombocytopenia according to the Common Terminology Criteria for Adverse Events (CTCAE version 4.0 ; 2010) (9). Radiotherapy was administered according to a 3-dimensional conformal technique. Regions of interest were contoured on CT scan images, acquired without contrast medium. Gross tumor volume measured $54.77 \mathrm{~cm}^{3}$, 


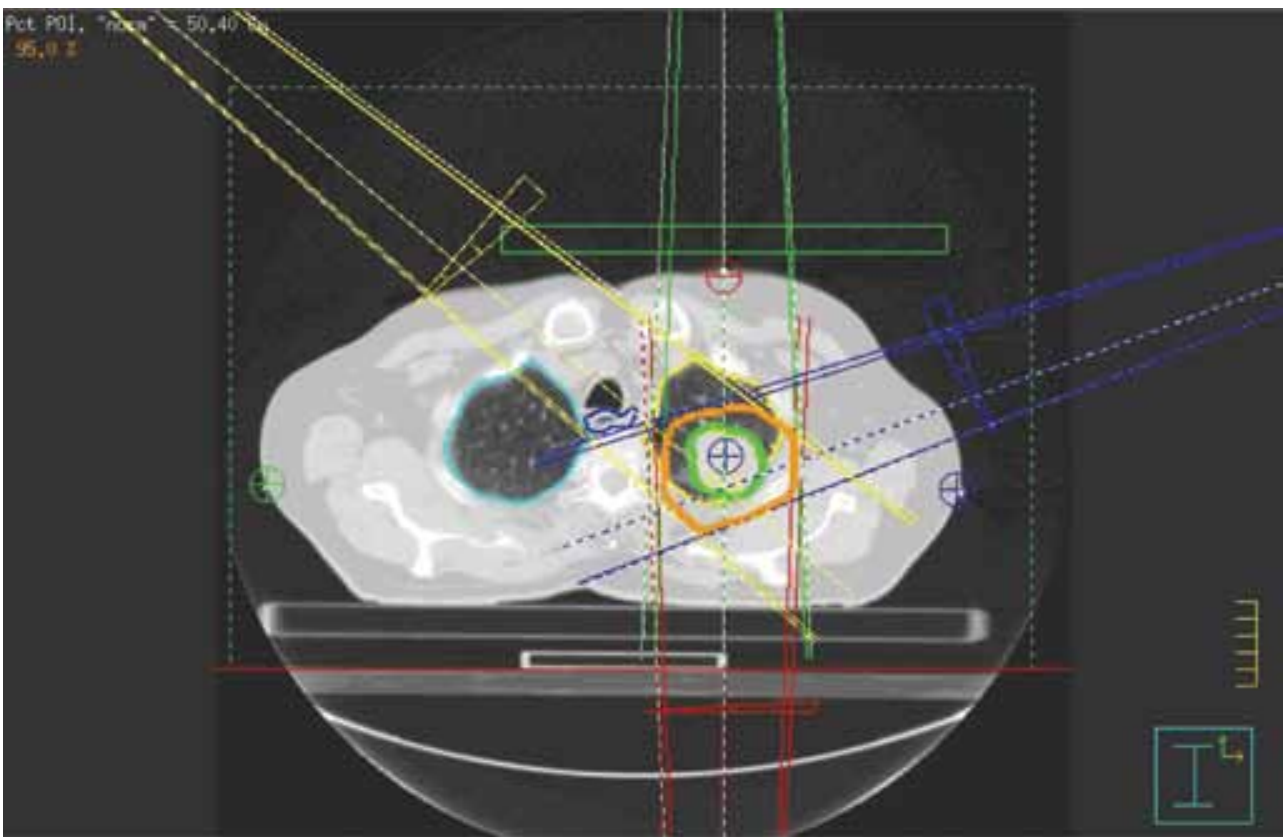

Fig. 1 - Treatment plan: isodoses distribution around neoplastic lesion.

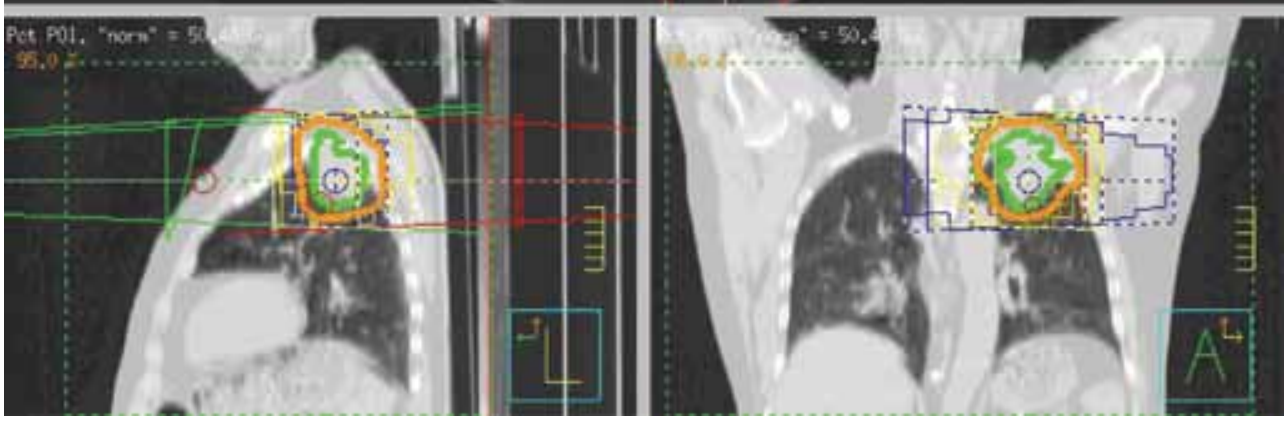

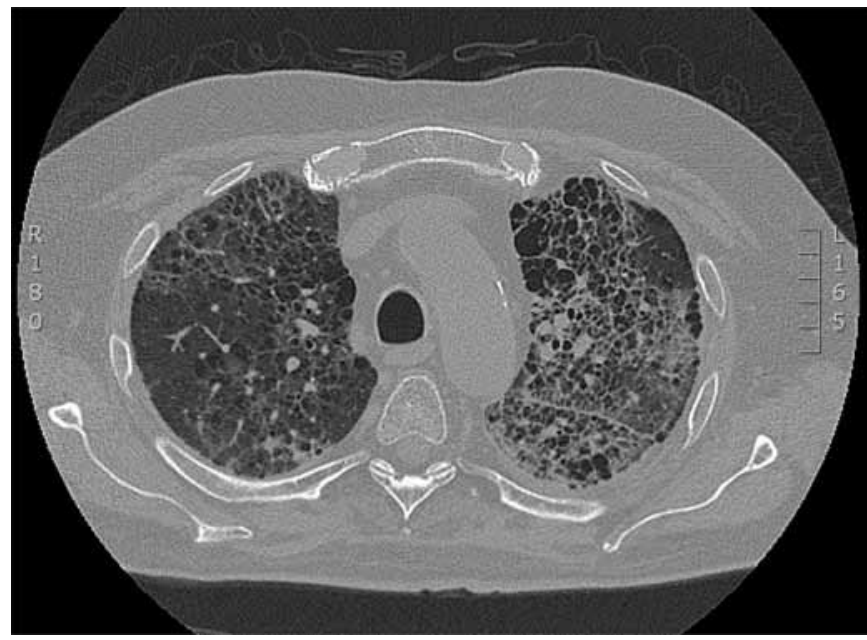

Fig. 2 - Diagnosis: Chest computed tomography scan with widespread ground-glass opacities and small nodular areas in the parenchyma.

+3 dyspnea and cough were reduced and $\mathrm{SatO}_{2}$ had risen to $90 \%$. The patient was more lucid, suggesting his mental confusion had been caused by hypoxemia. On day +7 , a chest $60 \%$ ) and methylprednisolone (40 mg 3 times daily). On day 

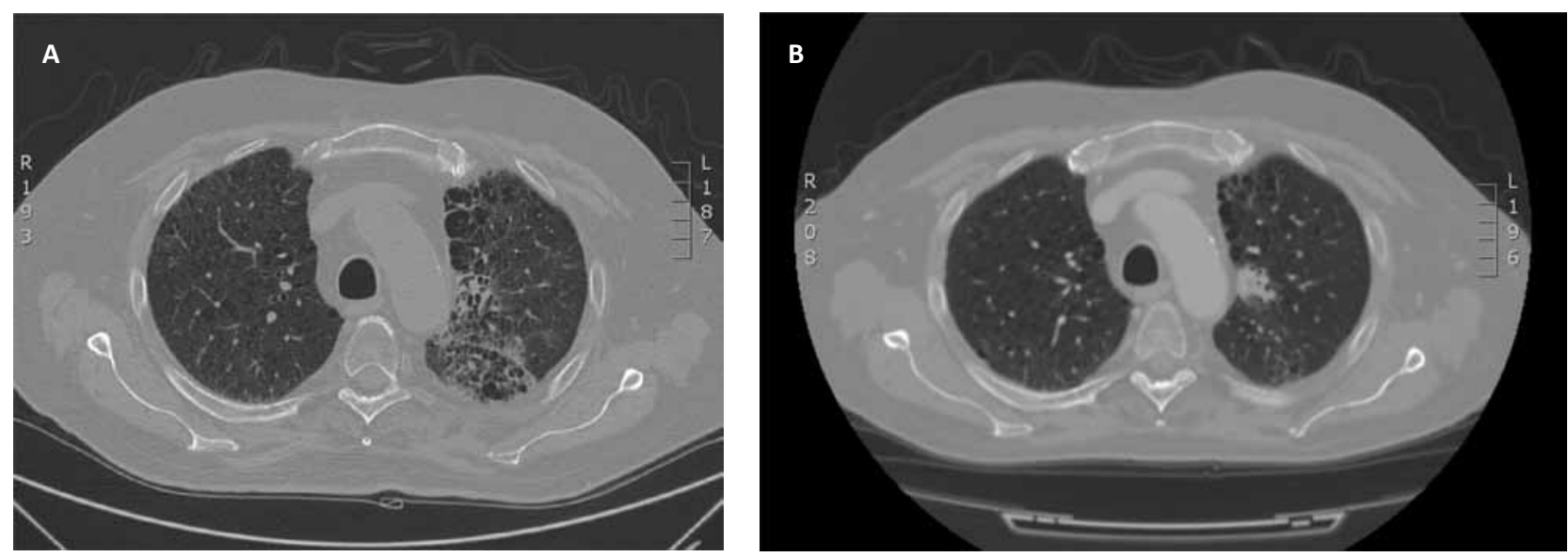

Fig. 3 - (A) 21 days after steroid treatment: chest computed tomography scan with minimal residual ground-glass opacities. (B) Three months after steroid treatment: chest computed tomography scan with no signs of bronchiolitis obliterans organizing pneumonia.

X-ray showed less reticular interstitial thickening. The primary tumor was unchanged. On day +21 , a chest CT scan visualized marked improvement in both lungs, with only minimal residual ground-glass opacities (Fig. 3A). Spirometry indicated $\mathrm{FEV}_{1}=52 \%$, SatO $_{2}=92 \%$.

The patient was discharged from the hospital. No surgery was scheduled because of his poor lung function. On day +90 , a CT scan showed no signs of interstitial pneumonia (Fig. 3B) but local and systemic progression of the primary tumor. Spirometry results were better $\left(\mathrm{FEV}_{1}=73 \%\right.$, SatO $=$ $94.5 \%)$. Corticosteroid therapy was continued up to 6 months. The patient died of disease progression 11 months after BOOP diagnosis.

\section{Discussion}

The most common side effect of RT to the lung is radiation pneumonitis with onset in irradiated parenchyma and risk linked to the dose delivered to the ascertained healthy lung volume (11).

We did not expect radiation pneumonitis to develop in our patient since the RT dose was administered to a restricted lung area and the RT plan met all lung dose constraints. Furthermore, chest X-rays and CT findings showed the affected areas extended beyond the area that had been irradiated. Consequently, symptoms, X-rays, and CT findings led us to diagnose BOOP and to start steroid therapy, to which the patient responded rapidly, confirming the diagnosis.

Bronchiolitis obliterans organizing pneumonia is a rare syndrome $(1,12)$. First described by Epler et al $(5)$ in 1985 in a heterogeneous group of patients, it is a flu-like illness with or without cough and bronchial crackles, lasting for 4-10 weeks $(3,4)$. As in our patient, chest X-rays and CT scans show, in both lungs, peculiar patchy areas of ground-glass densities and migratory, peripheral air-space infiltrates, extending to nonirradiated parenchyma $(1,5,6)$. Polypoid fibrino-inflammatory granulation tissue is also observed, extending from the bronchioles into adjacent alveoli, where pneumonia develops. Eosinophil, neutrophil, and lymphocyte counts are increased in bronchoalveolar lavage fluid. The etiology of BOOP is unknown. Although most cases are idiopathic, connective tissue disease (13), drugs $(8,14-17)$, infection $(18,19)$, toxic inhalation (20), and RT have been proposed as causes. In BOOP, RT $(21,22)$ is hypothesized to activate pulmonary lymphocytes, leading to immunologic and hypersensitivity reactions in the nonirradiated lung. As our patient actively smoked during radiation treatment, he was at higher risk than a nonsmoker of developing interstitial lung disease, but we are unable to link smoking with BOOP development.

The present case of BOOP after RT in a patient with lung cancer is unusual. The disease was almost exclusively reported in a very small minority of women who received RT after breast-conserving surgery $(1,2)$ in whom concomitant risk factors might be hormonal therapy and age over 50 years (1).

Although the incidence of BOOP may be low because lung cancer is less frequent than breast cancer, BOOP may be mistakenly diagnosed as the more frequently observed and better known radiation pneumonitis in patients with lung cancer, thus leading to an underestimated incidence. We agree with Crestani et al (3), who hypothesized in 1995 that BOOP development depended on how much pleural tissue was irradiated, which is a wide area in patients with breast cancer because RT uses tangential fields. Although this does not usually happen during irradiation for lung cancer, our patient received extensive pleural irradiation because the lesion was located in the lung apex.

As observed in our patient, BOOP responds well to systemic steroid therapy, which rapidly resolves symptoms and normalizes radiologic patterns. Even though BOOP and its adverse effects cannot be prevented, members of the multidisciplinary team who are involved in care of patients with lung cancer should be aware of the syndrome, recognize the signs on images, and realize prompt steroid treatment is urgently needed. Therapy needs to be protracted even for up to a year $(2,21)$, to reduce the risk of recurrence.

\section{Disclosures}

Financial support: None. Conflict of interest: None. 


\section{References}

1. Takigawa N, Segawa $\mathrm{Y}$, Saeki T, et al. Bronchiolitis obliterans organizing pneumonia syndrome in breast-conserving therapy for early breast cancer: radiation-induced lung toxicity. Int J Radiat Oncol Biol Phys. 2000;48(3):751-755.

2. Katayama N, Sato S, Katsui K, et al. Analysis of factors associated with radiation-induced bronchiolitis obliterans organizing pneumonia syndrome after breast-conserving therapy. Int J Radiat Oncol Biol Phys. 2009;73(4):1049-1054.

3. Crestani B, Kambouchner M, Soler P, et al. Migratory bronchiolitis obliterans organizing pneumonia after unilateral radiation therapy for breast carcinoma. Eur Respir J. 1995;8(2): 318-321.

4. Bayle JY, Nesme P, Béjui-Thivolet F, Loire R, Guérin JC, Cordier JF. Migratory organizing pneumonitis "primed" by radiation therapy. Eur Respir J. 1995;8(2):322-326.

5. Epler GR, Colby TV, McLoud TC, Carrington CB, Gaensler EA. Bronchiolitis obliterans organizing pneumonia. N Engl J Med. 1985;312(3):152-158.

6. Crestani B, Valeyre D, Roden S, Wallaert B, Dalphin JC, Cordier JF. Bronchiolitis obliterans organizing pneumonia syndrome primed by radiation therapy to the breast. The Groupe d'Etudes et de Recherche sur les Maladies Orphelines Pulmonaires (GERM“O” $\mathrm{P}$ ). Am J Respir Crit Care Med. 1998;158(6):1929-1935.

7. Murai T, Shibamoto $Y$, Nishiyama T, et al. Organizing pneumonia after stereotactic ablative radiotherapy of the lung. Radiat Oncol. 2012;7(1):123.

8. Ernesto $S$, Arpin D, Nesme P, Pérol M. Diffuse interstitial lung disease linked to vandetanib. Clin Lung Cancer. 2012;13(3): 236-238.

9. CTCAE v4.0. Available at: http://evs.nci.nih.gov/ftp1/CTCAE/ CTCAE 4.03 2010-06-14_QuickReference_5x7.pdf.

10. Gorter RR, Vos CG, Halmans J, Hartemink KJ, Paul MA, Oosterhuis JW. Evaluation of arm function and quality of life after trimodality treatment for superior sulcus tumours. Interact Cardiovasc Thorac Surg. 2013;16(1):44-48.

11. Tsujino $\mathrm{K}$, Hashimoto $\mathrm{T}$, Shimada $\mathrm{T}$, et al. Combined analysis of V20, VS5, pulmonary fibrosis score on baseline computed tomography, and patient age improves prediction of severe radiation pneumonitis after concurrent chemoradiotherapy for locally advanced non-small-cell lung cancer. J Thorac Oncol. 2014;9(7):983-990.

12. Miwa s, Morita S, Suda T, et al. The incidence and clinical characteristics of bronchiolitis obliterans organizing pneumonia syndrome after radiation therapy for breast cancer. Sarcoidosis Vasc Diffuse Lung Dis. 2004;21:212-218.

13. Balduin R, Giacometti C, Saccarola L, et al. Acute fibrinous and organizing pneumonia in a patient with collagen vascular disease "stigma". Sarcoidosis Vasc Diffuse Lung Dis. 2007;24(1):7880. Letter.

14. Ando M, Okamoto I, Yamamoto N, et al. Predictive factors for interstitial lung disease, antitumor response, and survival in non-small-cell lung cancer patients treated with gefitinib. J Clin Oncol. 2006;24(16):2549-2556.

15. Shogbon AO, Hap J, Dretler R, Dalvi AG. Cryptogenic organizing pneumonia during adjuvant chemotherapy with oxaliplatin, 5-fluorouracil, and leucovorin (FOLFOX) for colon cancer. $J$ Pharm Pract. 2013;26(1):62-66.

16. Fekrazad MH, Eberhardt S, Jones DV, Lee FC. Development of bronchiolitis obliterans organizing pneumonia with platinumbased chemotherapy for metastatic rectal cancer. Clin Colorectal Cancer. 2010;9(3):177-178.

17. Dahlqvist C, Fremault A, Carrasco J, Colinet B. [Obliterative bronchiolitis with organising pneumonia following FOLFOX 4 chemotherapy.] Rev Mal Respir. 2010;27(1):84-87.

18. Haroon A, Higa F, Hibiya $K$, et al. Organizing pneumonia pattern in the follow-up CT of Legionella-infected patients. J Infect Chemother. 2011;17(4):493-498.

19. Cunha BA, Syed U, Mickail N. Renal transplant with bronchiolitis obliterans organizing pneumonia (BOOP) attributable to tacrolimus and herpes simplex virus (HSV) pneumonia. Heart Lung. 2012;41(3):310-315.

20. Cheng TH, Ko FC, Chang JL, Wu KA. Bronchiolitis obliterans organizing pneumonia due to titanium nanoparticles in paint. Ann Thorac Surg. 2012;93(2):666-669.

21. Cottin V, Frognier R, Monnot H, Levy A, DeVuyst P, Cordier JF; Groupe d'Etudes et de Recherche sur les Maladies "Orphelines" Pulmonaires. Chronic eosinophilic pneumonia after radiation therapy for breast cancer. Eur Respir J. 2004;23(1):9-13.

22. Prakash UBS. Radiation-induced injury in the "nonirradiated" lung. Eur Respir J. 1999;13(4):715-717. 